\title{
Exploring barriers and facilitators to eating the Mediterranean diet in England: a qualitative study
}

\author{
G. Middleton ${ }^{1}$, R. Keegan ${ }^{2}$, M. F. Smith ${ }^{1}$, A. Alkhatib ${ }^{3}$ and M. Klonizakis ${ }^{3}$ \\ ${ }^{1}$ College of Social Sciences, School of Sport and Exercise Science, University of Lincoln, Brayford Way, Lincoln, \\ Lincolnshire, LN6 7TS, UK, ${ }^{2}$ Faculty of Health, University of Canberra, Bruce, Australia and ${ }^{3}$ Centre for Sport and \\ Exercise Science, Sheffield Hallam University, Sheffield, Yorkshire, S10 2BP, UK
}

Scientific guidelines and recommendations strongly encourage a Mediterranean-like dietary pattern for primary and secondary prevention of major chronic diseases ${ }^{(1)}$. In the United Kingdom (UK), several credible sources of dietary information have recommended consumption to the public (NHS Choices and Patient UK). The concept of how British citizens incorporate a Mediterranean diet (MD) into daily life (e.g. food availability, cost, personal preferences and preparation) has remained unexplored. Little is known on the pragmatic and cultural perspectives of adopting the MD in the UK. This study aimed to highlight the barriers and facilitators to eating the MD from a sample of participants who had recently attempted to implement the diet in the north-east of England.

The investigation recruited a sample $(n 11)$ of adult $(50-65 \mathrm{yrs})$ participants $(n)$ ) that had previously enrolled on an 8-week MD intervention study ${ }^{(2)}$. They were encouraged to attend this investigation with their co-habiting partner $(n 3)$. Semi-structured focus groups (3) were digitally recorded and transcribed verbatim; these allowed participants to discuss their experiences regarding 'barriers' and 'facilitators' whilst attempting the MD intervention. Additional field notes supported data collection. Data were analysed through a process of thematic content analysis from the concept-driven design ${ }^{(3)}$.

Key themes highlighted that participants enjoyed the pleasurable experience, reporting several physical benefits from adapting to this diet. Furthermore, they felt that the diet introduced a better quality of food, widened the food-horizon and allowed them to redefine cultural eating habits. Participants expressed difficulty in accommodating the diet with work and stress undermining adherence along with the pressures of time and family. They also suggested that it is difficult to purchase food items and regarded the British culture as non-conducive to this pattern of eating. The table illustrates views on how adherence to the MD could be improved:

\begin{tabular}{l} 
Participant suggestions to improve adherence \\
\hline Assist participants with the planning and organisation involved \\
Availability and suitability of further recipes \\
Supportive spouse/partner/family member \\
Social support opportunities with other participants \\
Recruiting participants who 'want' and 'commit' to changing diet
\end{tabular}

The data collected displayed the perceived enabling factors and apparent challenges when attempting to adhere this diet. This provides cultural information for the future development of appropriate and effective MD interventions with UK based participants.

1. Bach-Faig A, Berry E, Lairon D et al. (2011) Public Health Nutr 14, 2274-2284.

2. Klonizakis M, Alkhatib A, Middleton G et al. (2013) Clin Sci 124, 579-587.

3. Fade S \& Swift J (2010) J Hum Nutr Diet 24, 106-114. 